\title{
Crisis Team Setup for Better Improvisation
}

\author{
Jean-Jaques Kohler \\ Geneva School of Economics and Management, University of Geneva, Switzerland. E-mail: jean- \\ jaques.kohler@etu.unige.ch \\ Emmanuel Fragniere \\ School of Management, HES-SO Valais-Wallis, Sierre, Switzerland and University of Bath, Bath BA2 7AY, United \\ Kingdom.E-mail: emmanuel.fragniere@hevs.ch
}

\begin{abstract}
Major events and crises are becoming increasingly complex. In addition, emerging risk such as cyber risks, social engineering, social exclusion, must be addressed. On the other hand, we see that Business Continuity Plan protocols have changed little over time. They are based on logistical approaches that are mainly concerned with "physical" crisis management. In this paper, we want to emphasize the lack of scientific knowledge about the rules of composition of crisis teams and that a crisis team today has to deal with unforeseen crisis situations. In a recent research based on a qualitative survey of crisis experts and specialists, we developed the thesis that the constitution of crisis teams includes members that have very different experience profiles and furthermore not come from senior management positions. The reason for this is that most of these teams have to operate on an improvised basis today due to the complexity of modern crises. It is thus then important to set up an appropriate training and preparation phase once the crisis team is constituted. In this article, based on our field research and theory building we propose an original approach, adapted from Nonaka's SECI (Socialization, Externalization, Combination, Internationalization) model, to prepare the crisis team for better improvisation.
\end{abstract}

Keywords: crisis management, crisis committee members, improvisation, qualitative survey, resilience, orchestration.

\section{Introduction}

What are the important elements in the composition of a crisis team to better manage the unexpected? As part of this research, we propose to answer this question, which is crucial for the successful implementation of crisis resolution. Fragnière and Sullivan (2007) define a crisis as an event that puts strong, destabilizing pressure on organizations facing critical situations and subject to strong external and internal pressures. Responding to crises requires thus the ability to deal with unforeseen events and adapt to new conditions, including interconnection between different infrastructures, whether critical or not.

On the 11th March 2011, the Fukushima Daiichi nuclear power plant suffered major damage from the magnitude 9.0 earthquake and tsunami. Following this accident, some Swissbased companies decided to activate their business continuity plans and to establish their crisis committees. As per the pre-defined plans, local employees and their direct families should have been moved from Japan to other countries. It appeared that some employees or family members did not have a valid passport or simply did not accept to leave the country without their entire family putting the crisis management team under pressure to find solutions in a very short period of time.
On the 14th June 2017, a fire broke out the Grenfell Tower based in London, killing 72 people. It is considered as the worst loss of life to fire in UK domestic premises since the Second World War.

The phase 1 report of the Grenfell Tower Inquiry (see www.grenfelltowerinquiry.org.uk for latest developments) highlighted, among other things, that London Fire Brigade incident commanders were not trained to cope with the fire and there was no contingency plan for evacuation, failed to lift the "stay put" advice when the stairs remained passable, and also that communications systems failed and there were serious deficiencies in command and control. These two important events are good examples of unforeseen situations where the crisis management team must orchestrate a large number of heterogeneous actors in a very short time. In a recent research Kohler et al. (2020) revealed that the different crisis teams that performed the best were composed of members with different socioeconomic profiles and experiences and that, overall, these crisis teams were able to create a common vision based on an unstable situation. Consequently, in this paper, we propose a new approach for preparing the crisis team by relying on the SECI model (see Figure 1) developed by Nonaka et al., (2000) and in particular on "Socialization" (S from SECI, from tacit

Proceedings of the 30th European Safety and Reliability Conference and

the 15th Probabilistic Safety Assessment and Management Conference

Edited by Piero Baraldi, Francesco Di Maio and Enrico Zio

Copyright (C) ESREL2020-PSAM15 Organizers.Published by Research Publishing, Singapore.

ISBN/DOI: TBA 
knowledge to tacit knowledge) that typically corresponds to the improvisation phase of the crisis.

\section{Literature Review}

When a significant risk occurs, it is usually referred to as a disaster or a crisis. Fritz and Williams describe disasters and catastrophes as events for which a society is in serious danger and suffers such impacts and losses that the social structure is disrupted and the performance of all or some of the essential functions of the society is prevented. Fragnière and Sullivan (2007) define the crisis as an event that puts strong, destabilizing pressure on organizations facing critical situations and subject to strong external and internal pressures. Thus, the same event may be considered as a disaster or a crisis.

Park et al. (2013) highlight the importance of combining risk and resilience analysis, especially for complex systems. Indeed, when analyzing emerging hazards or threats, it is difficult to identify them because they are often unknown. They give as an example nuclear power plants or space programs where errors occurred in a way that would seem extraordinary, but which ultimately had similar origins: computer problems, errors in the interpretation of indicators, not taking into account alarms, bad decisions and communication problems between the different teams for example. It is interesting to compare the "resilience" and "risk" approaches. Resilience approaches require preparation for the unexpected, while risk analysis assumes that risks are known as explained by Mitchell and Harris (2012). The scientific literature on resilience is abundant (see for instance Fox-Lent et al. (2015), Aldrich and Meyer (2015), Meerow et al. (2016), Opdyke et al. (2017), Pescaroli et al. (2018), Curt and Tacnet (2018).

In the field of risk management, resilience is the ability of a system or company to cope with a disaster and recover quickly from the event. The term "resilience" has been used in physics for more than a century to describe the ability of a structure to absorb a shock or continuous pressure without breaking or being deformed. As early as 1973, Hollings (1973) redefined resilience in the context of ecology. Thus, Hollings defines resilience as the ability of an ecosystem to integrate a disturbance into its functioning without modifying its qualitative structure.

Responding to crises requires thus the ability to deal with unforeseen events and adapt to new conditions, including interconnection between different infrastructures, whether critical or not. This increasing complexity of interdependencies between different infrastructures often leads to the involvement of several actors in the management of a crisis that could affect one or the other of these infrastructures. Frykmer et al. (2018) investigated whether the ability to improvise collectively can explain, predict and be used to assess overall performance in crisis management. Following an exploratory study they conducted in the scientific literature on the term "improvisation", they found that existing scientific research was not sufficiently developed or detailed to achieve the objective of their analysis. Thus, the authors suggest that the links between improvisation and performance should be studied in order to better understand the effects of improvisation on collective performance in crisis management.

An element that should not be underestimated is the stress that could jeopardize the proper execution of the crisis management plan (see for instance Dilenschneider and Hyde, 1985). According to Perry (2004), crisis management exercises have a positive impact on the perception of knowledge among professionals and volunteers.

Our literature review in the field of crisis management did not allow us to find scientific knowledge about the typical composition of a crisis management team. Nevertheless, as noted by Mitroff et al. (1987) and Coombs (2019), the members of the crisis management team should possess a set of crisis management skills. Coombs (2019) described the profiles of the desirable and undesirable crisis team member in order to select the most competent crisis team. The desirable profile should have a high tolerance in ambiguity.

We however acknowledge that an improvisation phase in crisis management rely on tacit knowledge or know-how and that the members of the crisis committee will interact together relying on their own tacit knowledge.

According to the SECI model described by Nonaka et al. (2000), a newly created knowledge to be relevant must go through the following 4 steps of "knowledge transformations".

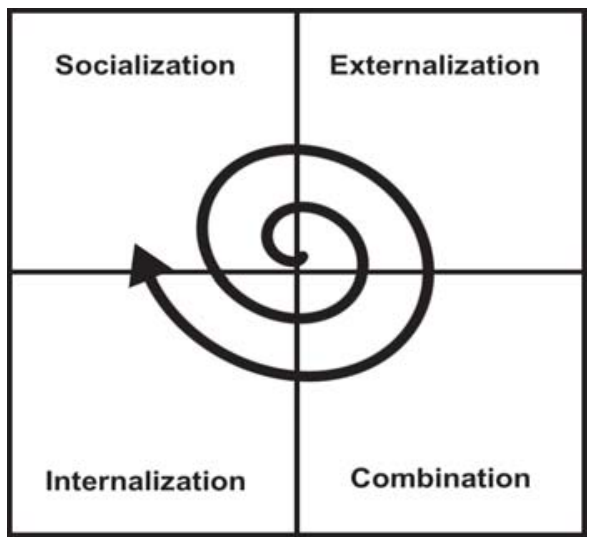

Fig. 1 - SECI model according to Nonaka et al. (2000) 
First, in the Socialization step (S of SECI), people are creating new tacit knowledge out of existing tacit knowledge.

Then, this new tacit knowledge is codified and becomes a new explicit knowledge. This step is called Externalization (E of SECI) and corresponds to what we call in general digitalization.

The third step is the Combination (C of SECI), it uses new explicit knowledge to produce new explicit knowledge. For instance, Artificial Intelligence (AI) can typically be considered as a Combination phase. The last step is the Internalization (I of SECI). It means that the new explicit knowledge produced by the Combination phase must be transformed into new tacit knowledge usable ultimately.

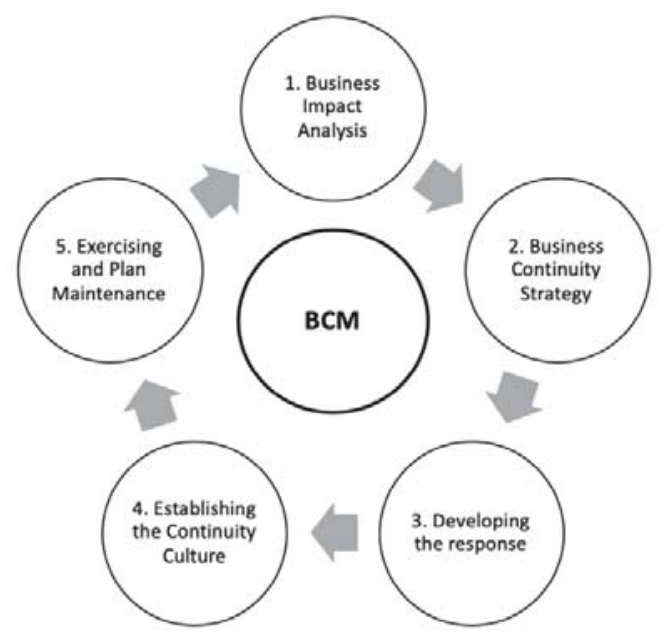

Fig. 2 - Business Continuity Management Model according to ISO 22301

To conclude this literature review, we show in Figure 2 the generic Business Continuity Management (BCM) model since it is an acknowledged reference in the field of business continuity management and in our theoretical development, we will rely both on this model and on the SECI model shown in Figure 1.

\section{Reflection on Crisis Team Composition}

It is thus essential that the members of a crisis committee can work together. Indeed, Ruelle (2012) characterizes crisis management as rapid decision-making in the presence of several choices, in a minimum of time. Consequently, planning a crisis committee must be a decision taken by top management before an event. This crisis committee must be composed of members with experience in crisis management and be able to manage situations where improvisation is required. The members of the crisis committee must be able to regularly train to deal with crisis situations. Most training scenarios focus on logistical or human risks. In our opinion, the risks related to new information technologies or those related to suppliers are not sufficiently developed. The development of new crisis management scenarios deserves particular attention, particularly in areas related to technological risks.

To be considered effective, Combalbert (2012) explains (see Figure 3) that a crisis team must be R.E.A.C.: Reactive, Effective, Adaptive, and Cohesive. Preparations and trainings help to increase the abovementioned characteristics.

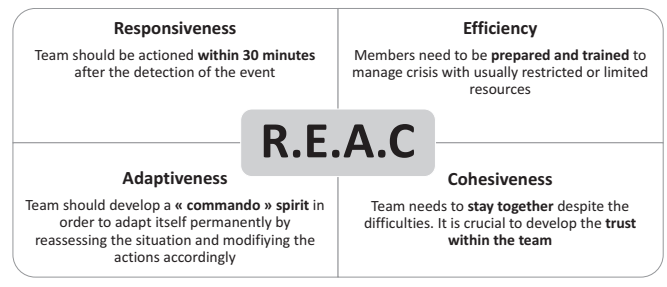

Fig. 3 - Characteristics of crisis committee by Combalbert (2012)

In an environment where companies are increasingly dependent on new technologies and interconnected with other companies, crisis management, and therefore the crisis committee, must evolve. This is why we believe that the crisis management of companies, private or public, highly dependent on new technologies and interconnected would deserve the full attention of top management but also researchers and experts to develop new methods and techniques of crisis management.

There is not a day in the press when a poorly resolved crisis management event goes unreported. We have therefore taken an interest in the composition of a crisis team though a qualitative survey (Kohler et al., 2020). To this end, we conducted semi-directive interviews to understand what were the important elements in the composition of a crisis team to better manage the unexpected. We present here a summary of the main results of this exploratory research. It appears that experience takes precedence over the hierarchical level of the involved individuals. It even seems preferable not to involve or at least to limit the number of members of the upper management. Although our study shows the benefits of simulations, stress test scenarios, the composition of the crisis team has to be better integrated especially to take into account emerging risks such as cyberattacks where improvisation plays a crucial role. 
- Crisis timeline: varied cases of crisis resolutions were reported mainly in the service sector (bank, insurance, fast-food, casino, municipality...)

- Crisis team: usually minimum size is 3 , and actors from same or different organizations but have never worked together before

- Identified weaknesses: focus on individual and personal interests vs overall crisis resolution, scenarios used for rehearsing not adapted, lack of training, top managers often in denial

- Identified strengths: unexpected strong dedication of people to solve the crisis, apparently varied profiles composing the crisis team is a plus

\section{Reflexion on BCM in the Context of Crisis Improvisation}

A fire drill is probably the best-known type of business continuity management, as everyone has at least once at an evacuation drill, if not as employees, then at least as elementary school students. Sprinklers, hoses and fire extinguishers are available in most buildings to prevent the spread of a fire. Even if a fire never happens, most people are required to follow the building's safe evacuation plan in the event of an alarm. Regular drills should be conducted to ensure the chances of success should a real evacuation be necessary. Even if simple procedures are designed, it can be fatal if they are not practiced regularly

Consider the following real-life example from a report that took place in France in the early 2000s A building was half occupied by a nursing school and half by a service company (see Fragnière and Sullivan, 2006). A fire broke out and all the staff had to evacuate the building. Only the nurses managed to leave the building calmly and safely. Employees of the service company were unable to follow the evacuation process, mainly because they had not taken the fire drills seriously. When a fire broke out, they panicked and attempted to escape by rushing to the exits. This only blocked the exits and prevented people from leaving the building. They all died.

Some will however say that it is impossible to predict how people will behave when a fire breaks out. and that it is impossible to make adequate emergency plans. If this is true, why were the nurses able to safely evacuate the building under the same circumstances? Nurses are trained by experts in emergency planning; they practice plans every day for dealing with emergencies. Therefore, the training of emergency plans is so important. We need to be like a "nurse" for our company and regularly train ourselves and our employees on how to behave in a case of emergency, whether it's a fire or a major disruption of computer systems or even many key people getting sick at the same time during flu season.

Business continuity plans should be in place to deal with all accepted risks, except perhaps those that are considered negligible, but especially those that are considered serious or catastrophic. That may seem like a lot of money and time spent on something that hopefully will never have to be used, but the cost of not having them when the risk arises would be even greater. Therefore, a costbenefit analysis should be done on a regular basis to determine which Business Continuity Plan (BCP) should be maintained or which new ones should be established.

These evacuation drills are therefore not to be taken lightly. They're here. They are mandatory and, as the previous little example shows, they are training to deal with any other business incident. Yes of course, ideally an exercise based on a scenario that corresponds to its own business context will allow us to repeat more specific and relevant routines. However, the thesis we put forward, which is based on a field survey, is that solidarity within the team, the personal commitment of the crisis team is more important than technical know-how to solve the crisis. Thus, we postulate that a simple drill exercise is a necessary but not sufficient condition to build a crisis team with the aim of improvising to deal with technical unknowns that probably could not have been foreseen in advance.

A crisis today corresponds to a situation where a tipping point is reached that prevents a company from functioning properly. A crisis often results from the most unexpected events, such as the sudden loss of key employees, unexpected lack of financial liquidity, reputation damage, operational failure or failure of strategic projects. The possible causes of a crisis are as numerous as the variety of business models, management models and organizations that exist around the world today.

The crisis can also be seen as an expression of risk. It means that the company has not achieved the expected profit or results despite the resources invested.

In response to a completely unforeseen crisis (e.g. a crisis for which no BCP has been or could have been established), emergency powers should first be introduced and reporting lines clarified. A person or small team should have full executive authority to manage the business during a storm. Those who have received extraordinary powers must be people that employees have trust and respect, people who have demonstrated their ability to swim, not to sink when they sink in troubled waters. These people must also have a thorough and profound knowledge of business. 
And they must communicate effectively in times of crisis. The company must speak with one voice.

Creating extraordinary powers is key to being able to respond quickly to critical issues. Managers must make decisions based on limited information. Does the company need to sell this type of business to get some liquidity? Must it require a high credit rate? Give up the key, expensive employees? Betting on the very existence of a new product?

In each of these results, this decision will certainly be unpopular with some employees and possibly investors. The survival of the company is at stake, usually not so many options, and they must be made by a trusted leader in a short period of time. Although these decisions may be made by some people, it is irresponsible to sacrifice the survival of the company, trying to make everyone happy.

BCM is a growing business, which started its operations largely as a result of the unjustified paranoia about the risk associated with the millennium $\mathrm{Y} 2 \mathrm{~K}$. But this really came to light after the terrorist attacks in New York and Washington, D.C. on September 11, 2001.

$\mathrm{BCM}$ can be considered as a subgroup of risk management. The common denominator of this practice is to help companies develop contingency plans in the event of major disruption of operations or environmental degradation. This means that a company must identify any type of accident or potential disaster, both internal and external, and develop an action plan for each of them that will allow them to continue operations despite the disruption, in a "degraded mode".

Both companies and organizations are becoming more and more networked. In addition, major events and crises are becoming increasingly complex. In addition, emerging risks: such cyber risks, social engineering, social exclusion, must be addressed. On the other hand, we see that BCP protocols have changed little over time. They are based on logistical approaches that are mainly concerned with "physical" crisis management. In this paper we want to emphasize the lack of scientific knowledge about the rules of composition of crisis teams and that a crisis team today has to deal with unforeseen crisis situations.

\section{A New BCM Model Based on Socialization Dedicated to Crisis Improvisation}

The concept of improvisation is linked to socialization (from tacit knowledge to tacit knowledge according to the SECI mode).

To develop our thesis, we rely on the theorical model called SECI by Nonaka (see Figure 4). There are 2 types of knowledge: explicit or codified knowledge and the tacit knowledge or know-how. According to this model, in order to create new knowledge, in our case to solve a crisis, we have to go through 4 phases: socialization: from tacit to tacit knowledge;

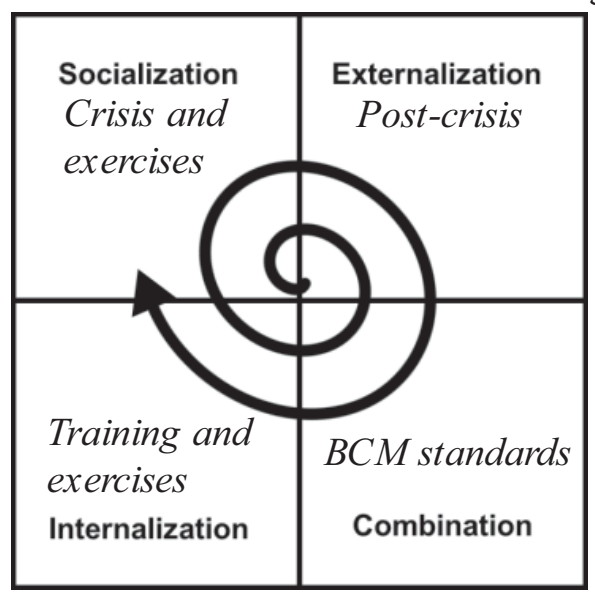

Fig. 4 - SECI model according to Nonaka et al. (2000), integrating the notion of crisis team improvisation role

externalization: from tacit to explicit knowledge; combination: from explicit to explicit knowledge; and finally internalization: from explicit to tacit knowledge. So when we solve a crisis, we are in a pure socialization phase, which we call "improvisation" in crisis management jargon. In order to work, the other phases must be carried out in a long preparatory phase. In the following subsections, we revisit the SECI model by adapting it to a new model of $\mathrm{BCM}$ for crisis improvisation.

\subsection{Socialization}

Socialization is a process of sharing experiences and is the way people interact with each other to solve a crisis. Socialization allows the team member to act quickly since they no longer have time to consult documents or explicit knowledge. They can get expert advice, but in this case it is an exchange of tacit knowledge. It also corresponds to learning through observation, imitation and practice, usually during a BCP simulation exercise. Here it allows for the acquisition of tacit knowledge shared among a group of people.

\subsection{Externalization}

Externalization is a process of articulating tacit knowledge into explicit concepts and thus a knowledge creation process involving the creation of metaphors, concepts, analogies, hypotheses or models created through dialogue or collective reflection. This phase is very important in a post-crisis phase where (see below) information is collected from crisis managers immediately after their involvement in a real crisis. 


\subsection{Combination}

Combination is a process of systematizing concepts into a knowledge system. It reconfigures existing information and knowledge into a useful toolbox for professionals and specialists. Outsourcing allows experts to codify this type of knowledge. For example, with respect to crisis management knowledge, standards are established that are intended to assist professional bodies. For example, ISO 22301 has been created for business continuity management knowledge and ISO 27031 for IT continuity and disaster recovery planning knowledge.

\subsection{Internalization}

Internalization is the process of incorporating explicit knowledge into tacit knowledge. It may involve learning-by-doing phases or, for example, the sharing of mental models or technical knowhow among practitioners. The professional standards produced during the Combination phase help crisis managers internalize new or updated knowledge and, through practice, transform it into new know-how that will be used in the case of a real crisis. This iterative process must continue indefinitely, as shown in Figure 1, so standards such as ISO 22301 and 27031 will have a real impact on the preparation of crisis teams. In general, $\mathrm{BCP}$ documents produced by organizations are very complex and specific, this approach should help crisis teams to better connect to BCPs so that they can use them as a "musical score" to achieve a harmonious and coordinated orchestration.

\section{Managerial implications for Crisis Preparation and Crisis Management}

In this section, we translate our findings proposed in the previous section into usable managerial precepts. We have deliberately used a didactic and accessible tone while relying on the major findings from our field study which are summarized below. However, we must also take into account the fact that all these tensions and pressures go through a process that we could call a life cycle of crisis stress intensity, as shown in

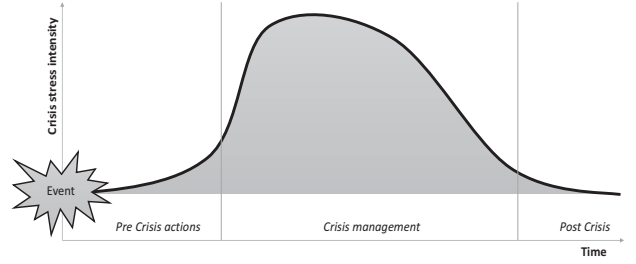

Fig. 5 - Life cycle of crisis stress intensity (adapted from Parsons and Pascale, 2007)
Figure 5. In fact, stress intensity increases slightly after the event (or major incident). Then, when we are in the phase of actual crisis management, this stress intensity reaches its paroxysm. And it then decreases significantly, only to gradually decrease in the post-crisis phase. This shows that preparation is crucial. It is incomprehensible that important management issues can be addressed at the height of a crisis, such as the formation of the crisis team.

\subsection{Pre-crisis action}

It is therefore absolutely necessary to set up a monitoring system already in the pre-crisis phase. This consists in a rigorous and systematic way of: identifying critical activities within the organization, as well as knowing its vulnerabilities (e.g. mapping of business risks), crisis potentials, careful monitoring of reports of undesirable events, managing complaints and disputes. It also means being attentive to weak signals such as rumors, to follow all types of media (classic and social networks). Indeed, these crisis threats must be detected as early as possible. The company's management should not be afraid to clarify subjects that would be taboo in the company.

\subsection{Building a crisis mechanism}

If we have enough information before a crisis occurs to make a diagnosis that the crisis is imminent, then we should set up a well-organized crisis unit as soon as possible. This is where BCM procedures and protocols are crucial. Crisis stress will quickly build up, so action plans (logbooks, procedures, protocols, activity plan, etc.) are needed to form the crisis unit and give it full power to orchestrate the crisis. When we talk about orchestration the word is not in vain. The members of the crisis unit will very quickly have to link and coordinate stakeholders and independent trades, who are not used to working together and who, above all, do not have the same work culture. It goes without saying that if this entire phase has not been prepared and trained through regular simulation exercises, no positive crisis resolution results can be expected! The interest of such training is that it is detached from the technical context of the crisis. It is essentially linked to the human factor and how these humans interact together. This is the interest of such training. They are not that expensive because they only involve a limited number of people and there is no need to integrate infrastructure elements (IT, building ...). These trainings are thus not intrusive and do not disturb the normal functioning of the company.

\subsection{Anticipating crisis communication}


Crisis communication must also be anticipated both internally and externally. It is again a question of accepting that the crisis unit will operate in improvisation mode and therefore that the "communication system" must be reliable but above all agile. Integrating into the crisis unit a professional who masters the new technologies and social networks is therefore crucial, while knowing the classic means of communication is crucial. Let's not forget that during the terrorist attacks in London on 7 July 2005, the police had switched off the mobile phone network, making any corporate crisis communication means based on this type of technology useless. To drive home the point, in the case of a major corporate crisis, we must also take into account the possibility of an electrical blackout, which in this case makes any technology totally obsolete.

\subsection{Post crisis}

Finally, post-crisis feedback is crucial regardless of the success or failure of its resolution. Unfortunately, little is known about the subject. Why certainly, because a managerial error will not be shouted from the rooftops. We could also mention the fact that each crisis is different and each time involves an extremely high level of technicality. But this is not the case in our approach. What interests us here is only a "qualitative" return on what happened at the level of the orchestration of crisis management by the team in charge of it. Therefore, the collection of information is relatively simple to set up. It involves semi-directive interviews and a synthesis and discussion as presented in our exploratory research about the composition of crisis team (see Kohler and Fragnière, 2020). The interview with the protagonists can be summarized by the following list of questions:

- What was your background at the time of the crisis?

- Were there any warning signs? How were they perceived? What were your first reflexes?

- What was the most difficult thing to do?

- Who was the most difficult to manage?

- Why was it the most difficult?

- What surprised you the most?

- What details were crucial in managing the crisis?

- How were the internal actors prepared?

- Can you identify the mistakes made on the basis of video recordings, press clippings, etc.?
- What changes should be made to your crisis plan?

- What was the impact of the crisis management on the image of the establishment?

- Relate all the stages of internal and external crisis communication.

Our final recommendation would be that a body specializing in crisis management should collect this type of feedback gathered during the postcrisis period. The cost of this activity would not be high; however, it would give a huge advantage that is not taken by any organization and any company that wishes to prepare for crisis management at the level of crisis unit training and training. What we need here are meanings as produced in ethnography (methodology used in Kohler and Fragnière, 2020), as well as elements that allow us to better contextualize the role of orchestration than that of a crisis committee in times of storm. All of this can be beneficial in times of calm, just before the storm!

\section{Conclusion}

Good business continuity management relies on simulations, stress test scenarios, standards, techniques and sophisticated technology. However, to successfully tackle a crisis, the composition of the crisis team must be better integrated, especially to take into account emerging risks such as cyber-attacks where improvisation plays a crucial role. In this article, we propose an adaptation of Nonaka's SECI model (Socialization, Outsourcing, Combination, Internalization) in order to prepare crisis teams to better interact in an improvised mode during a real crisis. A recent qualitative survey, which we conducted among professionals who have been involved in crisis management, showed that the quality of teamwork predominated over the technical aspects of $\mathrm{BCM}$ and therefore that the constitution of crisis teams must involve very different profiles. In this paper, we have shown how, that once the team has been set up on the basis of the proposed model, it is possible to train it to operate in an improvisational mode. In future research, we would like to apply this model to the training of crisis teams in parallel with large scale $\mathrm{BCM}$ simulation exercises.

\section{References}

Aldrich, D. P. and M. A. Meyer (2015). Social Capital and Community Resilience. American Behavioral Scientist 59 (2), 254-269. 
Combalbert, L. (2012). Le management des situations de crise: Anticiper les risques et gérer les crises. ESF Editeur.

Coombs, W. T. (2019). Ongoing Crisis Communication: Planning, Managing, and Responding ( $5^{\text {th }}$ ed.). SAGE Publications, Inc.

Curt, C. and J-M. Tacnet (2018). Resilience of Critical Infrastructures: Review and Analysis of Current Approaches. Risk Analysis 38 (11), 2441-2458.

Dilenschneider, R. L. and R. C. Hyde (1985). Crisis Communications: Planning for the Unplanned. Business Horizons 28 (1), 35-38.

Fox-Lent, C., M. E. Bates, and I. Linkov (2015). A Matrix Approach to Community Resilience Assessment: An Illustrative Case at Rockaway Peninsula. Environment Systems and Decisions 35 (2), 209-218

Fragniere, E., B. Nanchen, R. Ramseyer, P. Kuonen, B. Larpin, and P-Y. Roh. R. Wilk (2019). Fieldwork Analysis of Figurants' Involvement in an Airplane Crash Simulation in Order to Improve the Realism of the Exercise. 8th International Conference on Industrial Technology and Management (ICITM), 147-151.

Fragniere, E. and G. Sullivan (2006). Risk Management: Safeguarding Company Assets. Thomson Learning.

Fritz, C. E. and H. B. Williams (1957). The Human Being in Disasters: A Research Perspective. The ANNALS of the American Academy of Political and Social Science 309 (1), 42-51.

Frykmer, T., C. Uhr, and H. Tehler (2018). On Collective Improvisation in Crisis Management - A Scoping Study Analysis. Safety Science, Special Issue on societal safety, critical infrastructure reliability and related intersectoral governance, 110 (December), 100-109.

Ghauri, P. N. and K. Grønhaug (2005). Research Methods in Business Studies: A Practical Guide. Pearson Education.

Holling, C. S. (1973). Resilience and Stability of Ecological Systems. Annual Review of Ecology and Systematics 4, 1-23.

Kohler, J-J., E. Fragniere, and D. Konstontas (2020). On the Benefit of Mixing Varied Professional Skills to Better Handle Improvisation Phases in Crisis Management: A qualitative survey conducted in Geneva and Valais, Switzerland. 4th International Conference on System Reliability and Safety (ICSRS) (to appear).

Lagadec, P. (2013). Le Risque Technologique Majeur : Politique, Risque et Processus de Développement. Elsevier.

Meerow, S., J. P. Newell, and M. Stults (2016). Defining Urban Resilience: A Review. Landscape and Urban Planning 147 (March), 38-49.
Mitchell, T. and K. Harris (2012). Resilience: A Risk Management Approach. Overseas Development Institute, 1-7.

Mitroff, I., P. Shrivastava, and F. E. Udwadia (1987). Effective Crisis Management. Academy of Management Perspectives 1 (4), 283-292.

Nonaka, I., R. Toyama, and N. Konno (2000). SECI, Ba and Leadership: A Unified Model of Dynamic Knowledge Creation. Long Range Planning 33 (1), 5-34.

Opdyke, A., A. Javernick-Will, and M. Koschmann (2017). Infrastructure Hazard Resilience Trends: An Analysis of 25 Years of Research. Natural Hazards 87 (2), 773-789.

Park, J., T. P. Seager, P. S. C. Rao, M. Convertino, and I. Linkov (2013). Integrating Risk and Resilience Approaches to Catastrophe Management in Engineering Systems. Risk Analysis: An International Journal 33 (3), 356-367.

Parsons, G. D. and R. T. Pascale (2007). Crisis at the Summit. Harvard Business Review 85 (3), 80-89.

Perry, R. W. (2004). Disaster Exercise Outcomes for Professional Emergency Personnel and Citizen Volunteers. Journal of Contingencies and Crisis Management 12 (2), 64-75.

Pescaroli, G., R. T. Wicks, G. Giacomello, and D. E. Alexander (2018). Increasing Resilience to Cascading Events: The M. OR. D. OR. Scenario. Safety Science 110, 131-140.

Ruelle, S. (2012). Continuité d'activité et Gestion de Crise: De La Technique à l'humain. Securite et Strategie 10 (3), 32-40.

Saunders, M. N. K., P. Lewis, and A. Thornhill (2019). Research Methods for Business Students ( $8^{\text {th }}$ ed.). New York: Pearson. 